How to reference this article

Pietrzak, J. (2021). "Venezia è molto grande e bella" - due soggiorni della regina Maria Casimira d'Arquien Sobieska a Venezia e in Veneto negli anni 1699 e 1705. Italica Wratislaviensia, 12(1), 67-85. DOI: http://dx.doi.org/10.15804/IW.2021.12.1.04

\author{
Jarosław Pietrzak \\ Uniwersytet Pedagogiczny im. KEN w Krakowie, Polonia \\ e-mail: jaroslaw.pietrzak@up.krakow.pl \\ ORCID ID: 0000-0003-0341-2273
}

\title{
"VENEZIA È MOLTO GRANDE E BELLA"- DUE SOGGIORNI DELLA REGINA MARIA CASIMIRA D'ARQUIEN SOBIESKA A VENEZIA E IN VENETO NEGLI ANNI 1699 E 1705
}

\author{
"VENICE IS VERY GREAT AND BEAUTIFUL": \\ TWO RESIDENCES OF QUEEN MARIA CASIMIRA \\ D'ARQUIEN SOBIESKA IN VENICE AND THE VENETO \\ REGION IN 1699 AND 1705
}

\begin{abstract}
Queen Maria Casimira d'Arquien Sobieska had much contact with Venice. During Jan Sobieski III's lifetime, the queen kept in touch with Venetian diplomats (such as Angelo Morosini and Girolamo Alberti), imported luxury goods, imported luxury goods from Venice, and was interested in the local culture. The queen's departure from the countryside for the Eternal City in October 1698 determined that, at the very beginning — after crossing the Empire's border with Italy — she would meet the main cities of Veneto, Verona, Padua, and Venice. She returned to the Venetian Republic in 1705, when the queen, with papal permission, went to meet her daughter Teresa Kunegunda, Electress of Bavaria. In this article, several aspects of the queen's journeys are included, apart from the political matters already indicated. Firstly, the article details the ceremony of reception of the queen-widow by the authorities of the Serenissima, representatives of the Venetian elite, and envoys of foreign countries residing in Venice. Next, the article draws attention to the places that the queen visited and the piety she manifested, and it presents observations related to city life formulated directly by Queen Maria Casimira Sobieska and her courtiers, also in relation to the cities of Verona and Padua. Finally, the author of the article points out the queen's interest in the dramatic and musical repertoire presented on Venetian stages and in the whole of cultural life, including the carnival. To conclude, the author draws attention to the differences in the treatment of the royal status of the queen, depending on the political changes taking place in Europe and the reception of cultural and religious threads in the queen's life.
\end{abstract}

Keywords: Veneto, Queen Maria Casimira Sobieska, journey, carnival, ceremonial rules 
$\mathrm{D}^{2}$ urante il regno di Giovanni III Sobieski, i rapporti tra la Polonia e Venezia furono dovuti prevalentemente allo sforzo comune nella lotta contro le forze ottomane. Il momento culminante di questa alleanza fu il trattato della Lega Santa firmato a Linz il 5 marzo 1684, in cui la Confederazione polacco-lituana e la Repubblica di Venezia si unirono al Papa e all'imperatore romano (cf. Setton Kenneth, 1991, passim; Jačov, 2003, passim; Platania, 2010, passim). Venezia fu un alleata della Polonia. Al fine di rinforzare l'alleanza Giovanni III Sobieski propose perfino di mandare suo figlio primogenito, il principe Jacopo, a Venezia con la missione di partecipare nella spedizione anti-turca, anche se questi progetti non furono realizzati (cf. Giovanni III Sobieski a Kazimierz Bieliński, Varsavia, 25 VI 1688, AGAD, APP, rif. 51, k. 29r-4r.). I tre diplomatici veneziani: Alvise Morosini, il quale nel 1678 insieme al nunzio pontificio e il ministro dell'imperatore, portò ad un cambio di rotta nella politica estera polacca, strappando la corte dall'influenza francese (Wójcik, 1976, passim; Platania, 1998, pp. 242-268), Giacomo Cavanis (Platania, 2007, pp. 293-313) e Girolamo Alberti, che nel periodo 1684-1696 patrocinò l'alleanza, godevano di un grande rispetto della copia reale negli anni 1685-1699 (Komaszyński, 1990, pp. 109-115).

***

Il presente testo si pone l'obiettivo di parlare di due soggiorni di Maria Casimira d'Arquien Sobieska a Venezia e nel Veneto negli anni 1699 e 1705. Il passaggio di Maria Casimira, vedova di Giovanni III, attraverso i territori della Repubblica di Venezia verso la Città Eterna all'inizio del 1699, ebbe un carattere particolare. La regina polacca lasciò la Repubblica di Polonia dopo la perdita delle elezioni che privò suo figlio Jacopo della possibilità di ottenere la corona (cf. Komaszyński, 1984, pp. 213-222; Skrzypietz, 2011, pp. 247-345). Il disordine politico derivante dal mancato riconoscimento della maestà di Federico Augusto di Sassonia da parte dei sostenitori del candidato francese, il principe Francis Louis de Bourbon-Conti, accelerò la decisione di partire. Tuttavia, questa decisione richiedeva il consenso della Camera dei deputati della Repubblica di Polonia e di garantire il reddito e i crediti della regina ve- 
dova (Maria Casimira d'Arquien Sobieska a Pompeo Scarlatti, Varsavia, 29 I 1698, ASC, FS, n 1187, n.n.; M.C. d'Arquien Sobieska a Stanisław Antoni Szczuka, b. m., 11 IX 1698, AGAD, APP, segn. 164, pp. 84-86). L'invito del papa Innocenzo XII rivolto alla regina Maria Casimira per partecipare alle celebrazioni del giubileo straordinario dell'anno 1700 diventò un pretesto molto comodo (Bernardi, 1699, pp. 261-262). Tuttavia, bisogna ricordare che la regina, politicamente molto attiva, non volle essere privata dell'impatto effettivo sulle cose e accontentarsi del suo status di magnate. Lo fece notare più volte nella sua corrispondenza: "Devo conservare la mia posizione di regina con la stessa dignità che ebbi all'inizio e per fare ciò ho solo il mio patrimonio [...]. Sono sempre la stessa regina" (M.C. d'Arquien Sobieska a Jacopo Ludovico Sobieski, Roma, 1 IX [1703], NGAB, f. 695, op. 1, n² 272, k. 161r.). Un'altra volta, scrivendo al figlio, esprimeva le sue preoccupazioni: "Immagina chi sarei in Polonia! Costretta a stare a Lwów o a Jaworow, dove la maggior parte di quelli che si dichiarano nostri amici cercherebbero di venire, per non suscitare sospetti del re che li aveva già presi in giro quando soggiornavo a Varsavia" (M.C. d'Arquien Sobieska a J.L. Sobieski, [Roma], 31 X [1700], NGAB, f. 695, op. 1, n² 258, k. 33v.-34r.). Questo dimostra quanto forte fu il pericolo di degrado che la regina cercò di evitare.

Il viaggio a Roma della regina iniziò il 2 X 1698. Maria Casimira si mise in viaggio con un numeroso e ricco corteo. Il 29 ottobre, il corteo lasciò i confini della Repubblica di Polonia ed entrò in Silesia, appartenente all'imperatore, per poi proseguire passando per Opava, Olomuc, Reichenhall e Trento verso l'Italia (Komaszyński, 1984, pp. 214-215).

La regina decise di non attirare l'attenzione e di non fare ingressi ufficiali nelle città visitate. Forse il fatto di nascondersi sotto il nome di contessa di Jaworow fosse dettato dalla voglia di evitare spese di rappresentanza, investimenti nel vestiario sfarzoso per se stessa e per i cortigiani, carrozze decorate etc. Inoltre, essere in incognito le assicurava l'immunità e permetteva di inserirsi con discrezione nella società di corte per conoscere l'entourage del sovrano e le relazioni tra di loro (Bély, 1999, pp. 469-470; Langen, 2002, pp. 145-155). Come constatò lei stessa: 
Che cosa ha a che fare il fatto che sono cognito con il fatto che succedono degli errori? [...] Inoltre, essere in incognito aiuta in tutto. Uno smette di essere un principe polacco quando non usa il proprio cognome e mette il proprio servitore sul posto più importante durante una passeggiata in carrozza. (Jan Kosmowski a Alessandro Sobieski, Verona, 9 I 1699, BUW, segn. 76, k. 233v.-234r.)

Seguendo questo principio la regina continuò a rifiutare di far celebrare la sua persona, il che non fu per niente facile prendendo in considerazione il suo status reale. Questa difficoltà fu accentuata inoltre dall'incontro con i delegati della Repubblica di Venezia già a Innsbruck (ibid., k. 233r.). Il compito degli emissari consisteva nel guidare la comitiva attraverso i passi del Tirolo verso Borghetto sull'Adige, la prima località soggetta alla sovranità della Serenissima, e poi più avanti verso Verona:

Lì al confine, in principio, il governatore di Verona accolse Sua Maestà la Regina con grande onore. Subito dopo il nuovo ponte di barche fatto costruire di proposito dalla Repubblica per Sua Maestà la Regina fu predisposto un pernottamento. Dapprima, ci fu un banchetto solenne nella prima cittadina italiana dopo il ponte. Tuttavia, Sua Maestà non si sedette a tavola dicendo "che io vengo in incognito e non accetto onori così grandi". Sua Maestà mangiò le sue pietanze in modo comune. Tutta la gente veniva trattata solennemente dal più grande al più piccolo. Portavano un enormità di vini e di altre cose. L'indomani, venne da Sua Maestà il suddetto governatore chiedendo di consentire ad un discorso publice a Verona al che la Regina non volle acconsentire in nessun modo. (ibid., k. 233v.-234r.)

Kosmowski descrisse in questo modo l'accoglienza della regina nella villa Nichesola a Ponton appartenente al senatore Piero Mocenigo, che meravigliò Maria Casimira con la sua posizione incantevole e con delle magnifiche decorazioni scultoree (Conforti, 1988-1989/1989-1990, pp. 65-124).

La richiesta della regina di rimanere in incognito fu prontamente respinta dagli ospiti. Da quel momento in poi, Sobieska non rifiutò più di accettare l'invito ufficiale da parte del senato per fare un ingresso pubblico a Venezia. 
L'accoglienza in pompa magna dell'8 gennaio 1699 a Verona fu il primo grande e solenne evento di benvenuto della regina in terra italiana (cf. Komaszyński, 1988, pp. 153-163; Platania, 1988, pp. 165-181; Pietrzak, 2018, pp. 70-88). Le notizie dei giornali riferivano il grande omaggio reso alla vedova del re Giovanni III dai senatori della Repubblica presenti e il passaggio della regina sotto la Porta di San Zeno, illuminata dalle torci e ornata di raffigurazioni allegoriche delle virtù, verso il Palazzo del Capitanato (Trattamento fatto alla Regina di Polonia da Veneziani a Verona, B.A.V. FCh, Cod. M.V.V., k. 207r-208v.; Avviso di Venezia del 10 febbraio 1699, AAV, FA, vol. 64, pp. 155/b e 52/a; Platania, 1989, p. 289; Lewański, 1984, pp. 27-32). Il giorno successivo, alla regina vennero presentati degli ospiti illustri. Davanti al cospetto della regina si presentarono con i loro omaggi il governatore Francesco Vendramin e sua moglie Moceniga Mocenigo, che sfoggiavano delle vesti meravigliose e sfarzosi gioielli, principe di Mantova e Monferrato Ferdinando Carlo Gonzaga, Rinaldo Cardinale d'Este e Francesco Farnese principe di Parma e Piacenza (Francesco Vendramin a M.C. d'Arquien Sobieska, Verona, 22 I 1699, ASC, FS, busta 1190, fascicolo 4, s.p.). All'udienza seguì la visita della città e della cattedrale dove fu introdotta dal vescovo eletto Gianfrancesco Barbarigo (Biancolini, 1750, p. 389; Bassani, 1700, p. 70). Invece per concludere, fu organizzato un ballo con la partecipazione di quasi duecento dame e cavalieri. In segno di gratitudine, la regina: “[...] chiese alle sue dame d'onore e ai cavalieri polacchi di presentare alcuni balli alla Rutena, che tuttavia non risultarono molto raffinati in quanto gli strumenti non trasmettevano bene le melodie dei balletti" (Bassani, 1700, p. 71), il che rispecchiava le differenze dei gusti musicali e dell'ideale del decorum.

Andando al cuore della Serenissima, per attraversare il percorso fluviale tra Verona e Padova (Bassani, 1700, p. 72; Wrana, 2019, pp. 337-342) la regina salì su un'imbarcazione magnifica chiamata burchiello:

A un miglio di strada da Venezia incontrammo una barca nera di velluto dove sedevano due senatori veneziani [Vittore Zane e Giovanni Pasquali] in abiti in raso rosso lavorato in una strana maniera. In seguito, si avvicinarono all'imbarcazione di Sua Maestà e salirono sulla suddetta imbarcazione. Avendo chiesto ai barcaioli che trasportavano Sua Maestà di fermarsi, fecero 
allora i complimenti da parte del loro principe [Doge Silvestro Veliero] e di tutto il senato. Pregarono S.M. la regina di voler accettare l'accoglienza pubblica a Venezia e di acconsentire di soggiornare nel palazzo indicato dalla Repubblica di Venezia. Infine, chiesero che permettesse di fornire approvvigionamenti per lei e per tutta la corte. (J. Kosmowski a A. Sobieski, Venezia, 24 I 1699, BUW, segn. 76, k. 237v.-238r.)

Notiamo che su iniziativa del Comune di Padova e degli amministratori del santuario, si è deciso di addobbare la basilica e allestire un dignitoso ambiente musicale per salutare degnamente la regina. Il solenne corteo giunse a Padova nella notte del 13 gennaio 1699, alla luce di innumerevoli fiaccole e accompagnato da un gruppo di cavalleria prestato dal capitano Agostino Sagredo. Alle porte di Savonarola la regina si trasferì in lettiga e, accompagnata dalla sovrintendente della casa di corte e dal padre della regina, entrò nel centro cittadino, con gli applausi della folla in suo onore. La regina viveva nella Casa degli Specchi, nota anche come Palazzo Bassano, appartenente alla famiglia del cronista del suo viaggio a Roma e al canonico di Warmia (Ferrari, 1868, pp. 31-60). Per questa occasione la facciata del palazzo fu illuminata di luce abbondante e decorata con fiori. Il giorno successivo, Maria Casimira ricevette doni dalle autorità cittadine, costituiti da 78 vassoi con uccelli, selvaggina, frutta candita, conserve, tartufi, erbe aromatiche, carne e 12 scatole di liquori. Subito dopo, la vedova di Giovanni III, vestita di un mantello d'oro e adornata di gioielli, si recò nella Basilica di S. Antonio. Dopo aver ascoltato la santa messa con l'accompagnamento della musica, la regina visitò le reliquie del santo a cui rese omaggio. La regina proseguì la giornata con le visite di cortesia del capitano della città, accompagnata dalla nobiltà e dal vescovo Giorgio Cornaro. Maria Casimira visitò ancora una volta il tempio sacro il giorno successivo e pregò di nuovo sulla tomba del santo francescano (Lenart, Wrana, \& WojtkowskaMaksymik, 2019, pp. 361-362). La sera dello stesso giorno, in onore della regina, il Capitano Sagredo tenne un sontuoso ballo nella sala di lettura pubblica del Palazzo dei Capitanato. Maria Casimira, invece, si rifiutò di partecipare ai balli, accontentandosi del ruolo di osservatrice della commedia della nipote, chiamata anche Maria Casimira (ibid., s. 361-362). L'ultimo giorno, la regina iniziò i preparativi per partire per 
Venezia. La regina, per la terza e ultima volta, andò a messa a St. Antonio e poi al Monastero di Ognissanti, dove le furono mostrate le reliquie della Beata Elena Enselmini (Diario di quanto è seguito in Padova nella permanenza fattavi dalla Reale Maestà di Maria Casimira Regina di Polonia (Lenart, Wrana, \& Wojtkowska-Maksymik, 2019, pp. 362-364).

All'arrivo a Venezia, fin dall'inizio la regina fu preoccupata e amareggiata per il suo alloggio perché fu: "[...] in un posto molto stretto, scomodo e maleodorante" (J. Kosmowski a A. Sobieski, Venezia, 24 I 1699, BUW, segn. 76, k. 238v.-239r.). I cortigiani della regina, a cui venne negata l'ospitalità in città, sperimentarono delle difficoltà assai più grandi. Solo dopo l'intervento della regina e del nunzio apostolico Agostino Cusani, riuscirono a trovare la sistemazione nelle case dei Veneziani. Questa situazione fu dettata dal cerimoniale e dal fatto che la regina venne considerata una persona privata fino all'udienza davanti al Doge, ai consigli e al senato della Repubblica. Dal giorno dell'arrivo della regina, cioè dal 19 gennaio, passò una settimana prima che fosse organizzata la cerimonia ufficiale di benvenuto al Palazzo dei Dogi. La regina ricevette dal Doge e dalla Repubblica un ricco dono che consisteva in 150 catini d'argento pieni di diversi zuccheri, confetture, frutta candita, candele di cera, pietanze ecc. che dovevano costituire l'approvvigionamento del suo seguito (cf. Rovere, 1704, p. 140; Urban, 2007, pp. 84-85). Maria Casimira fu condotta al cospetto del Doge Silvestro Valiero e del Maggior Consiglio insieme a dieci dame e altrettanti cavalieri. Solo quattro giorni dopo, fu ricevuta dal Cancelliere Grande della Repubblica Pietro Businelli e dai senatori. Durante questo incontro fu pronunciato l'elogio della regina (Avviso di Venezia 7 II 1699, AAV, FA, vol. 64, p. 96; J. Kosmowski a A. Sobieski, Venezia, 25 I 1699, BUW, segn. 76, k. 247r.). Queste circostanze cambiarono sensibilmente la condizione della regina che da quel momento in poi fu trattata come ospite ufficiale. Per questo motivo, fu deciso di fornirle una nuova residenza più comoda e più consona ai requisiti del protocollo. La regina e il suo seguito furono sistemati nel Palazzo dei Foscari (Ca' Foscari) collocato nel sestiere Dorsoduro. Probabilmente questa sistemazione non fu casuale visto che nel mese di giugno del 1574, gli appartamenti del palazzo ospitarono un compatriota della regina, il re della Polonia 
Enrico di Valois fuggito di nascosto dalla Polonia per prendere la corona francese (Tassini, 1872, pp. 284-286).

Nei giorni successivi, la regina ricevette illustri ospiti in base al principio di precedenza. Il primo a farle visita fu il nunzio apostolico a Venezia, Agostino Cusani. Un testimone di questo incontro riportò: "Allora, le fece una benedizione e Sua Maestà gli rispose in francese dopodiché portarono le sedie e la Regina si sedette. Egli conversava con Sua Maestà sempre in italiano e lei gli rispondeva in francese. Comunque, non so se egli capisse la Regina, perché non conosceva una parola di francese. Rimase un'ora e se ne andò" (J. Kosmowski a A. Sobieski, Venezia, 24 I 1699, BUW, segn. 76, k. 239v.-240r.). L'udienza del patriarca di Venezia, Giovanni Alberto Badoer, si svolse con le stesse modalità. Altri due incontri furono molto più importanti per la regina. Il primo lo trascorse in una piacevole conversazione di ben due ore con il console francese Guillaume Le Blond. L'uomo diplomatico parlò dell'inizio della sua carriera, quando era a Costantinopoli come rappresentante del re di Francia nel 1654, dove ebbe l'onore di assistere all'entrata in città dell'ambasciatore polacco Mikołaj Bieganowski accompagnato dal sovrintendente di Jaworow Giovanni Sobieski che spiccava per educazione e conoscenza di lingue straniere. L'incontro che influenzò ancor di più la vedova di Giovanni III fu quello con il padre Marco d'Aviano, il patrono spirituale della campagna di Vienna. Nel 1683, il cappuccino rimase a Vienna assediata dai Turchi come inviato del papa Innocenzo XI presso l'imperatore e sosteneva con la preghiera tutti quelli che ci combattevano, incluso il re di Polonia (cf. Cavazza, 2007). "L'uomo di Dio", come lo chiamava Giovanni III Sobieski, accolse la regina il giorno successivo al suo arrivo, cioè il 21 gennaio 1699, porgendole le sue condoglianze: "v'andò il martedì di mattina, che fu ricevuto al lato con tanta distinzione ch'ella hebbe a dire al padre, che non haveva ricevuta tanta consolazione dopo la morte del suo marito. Si trattenne con Sua Maestà lo spazio di due ore, sempre in discorsi spirituali, e quando partì li promise che sarebbe venuta al convento ad ascoltare la sua messa e comunicarsi" (Platania, 1986, pp. 11-23). La liturgia di cui si parla nella citazione sopra riportata ebbe luogo nella chiesa del Santissimo Redentore. Durante la messa, il cappuccino diede la 
sua benedizione alla sovrana, il che la commosse: "Mio figlio carissimo, questo già basta alla persona che domani dovrà ricevere la Santa Comunione dalle mani del padre d'Aviano il quale venne vedermi e si ricordò pure di Te. Mio carissimo figlio, ti ho raccomandato alle sue preghiere, così come al principe, a tua moglie e a mia nipote" (M.C. d'Arquien Sobieska a J.L. Sobieski, a Venezia, 24 I 1699, NGAB, f. 695, op. 1, $\mathrm{n}^{\circ} 260$, k. 26r.-26v.) La seconda visita che ebbe luogo il 25 gennaio, fu invece di carattere più privato:

alle 19 hora, ma non con tutta la sua corte havendo solamente condotte due sole dame d'onore, quattro damigelle et alcuni gentiluomini et altra servitù, fu ricevuta come la prima volta [...] se il tempo l'havesse permesso, perché la voce sparsa che la regina doveva ritornare al Redentore e far le sue divotioni e niuno si sarebbe mai pensato che per essere il cielo nuvoloso e che il vento faceva fluttuare il canale, uscire di casa, ma la sua pietà supra ogni ostacolo e confusione di molti”. (Platania, 1989, pp. 294-295)

Dunque, per la regina l'incontro con il frate cappuccino fu una questione di prima importanza. La regina lo trattava come se fosse "una reliquia viva", oltre che testimone di una grande vittoria e guida spirituale di Giovanni III. Maria Casimira manifestava la sua devozione frequentando diverse chiese e conventi, il che consolidò la sua reputazione di sovrana pia e da seguire come modello di vita tra gli abitanti di Venezia. La regina visitò tra l'altro la chiesa dei Padri Teatini il giorno della festa di Purificazione della Beata Vergine Maria, il convento dei Padri Carmelitani dove adorava il Santissimo Sacramento e il monastero delle Benedettine di San Lorenzo (cf. Avviso di Venezia, 7 II 1699, AAV, FA, vol. 64, p. 96). I suoi pellegrinaggi furono inoltre un'eccellente occasione per visitare la città e per incontrare gli aristocratici veneziani, mentre la visita nella vetreria dell'isola di Murano fu di carattere più conoscitivo.

L'arrivo di Maria Casimira d'Arquien Sobieska a Venezia coincise con il famoso carnevale, organizzato sotto il segno della dominazione dei gusti francesi, di cui gli artefici furono Jean-François Regnard e André Campra (Duron, 2010). Famosa per il suo amore per le feste e per il patrocinio di manifestazioni musicali e teatrali, la regina partecipò 
a numerose feste e assistette alle rappresentazioni teatrali in suo onore e dedicate alla gloria del suo defunto marito (Platania, 1989, p. 292; Bassani, 1700, p. 70). Il 21 gennaio, visitò il Teatro di SS. Giovanni e Paolo per vedere uno spettacolo. Il giorno dopo si recò al teatro più glorioso di Venezia cioè il Teatro S. Giovanni Grisostomo dove assistette all'opera musicale Lucio Vero con il libretto di Apostolo Zeno:

Danno spettacoli in ben cinque posti diversi ma Sua Maestà frequenta di più il Teatro di San Grisostomo perché lì, il teatro è bello e gli attori così bravi che non puoi vederne né sentirne di migliori. Che belle voci delicate delle dame che ci sono e che begli abiti ricchi che portano. (J. Kosmowski a A. Sobieski, Verona 24 I 1699, BUW, segn. 76, k. 240v-241r.; cf. Markuszewska, 2012, p. 68)

Si presume che Marc'Antonio Ziani abbia composto e presentato l'opera Teodosio nel teatro di San Cassiano espressamente per la visita della regina (Selfridge-Field, 2007, p. 236). Maria Casimira fu oltremodo emozionata ricordando l'entrata al teatro d'opera:

$\mathrm{Ci}$ sono quattro teatri d'opera e quattro teatri di commedia - sono andata ad assistere a due opere liriche, molto buone, questo significa che qui, ci sono ben otto teatri gremiti di gente elegante. In tutti i loggioni siedono delle belle dame con cavalieri, la maggior parte porta le maschere. Il signor Foscarini fu con me nel loggione, in maschera. [...] Ho capito che questa città è una meraviglia del mondo. (M.C. d'Arquien Sobieska a J.L. Sobieski, da Venezia, 24 I [1699], NGAB, f. 695, op. 1, n² 260, k. 23v.)

La presenza al teatro dava l'occasione di incontrare la gente importante. Maria Casimira sfruttò quest'opportunità per vedere nuovamente il principe di Mantova e per conoscere l'erede al trono di Danimarca, il principe Federico della dinastia Oldenburg. Delle conversazioni e gentili inchini portavano in seguito a comuni festeggiamenti e a giochi d'azzardo. La regina apprezzava in particolare la bassetta. 
Durante il suo soggiorno di quasi venti giorni a Venezia, la regina fu meravigliata da questa città. Lo splendore di cui fu circondata le diede il senso di trionfo e di magnificenza. La regina non mancò di sottolinearlo con grande piacere durante il suo viaggio verso Roma: “[...] mentre proseguo il mio viaggio verso Roma passando per Ferrara e Bologna, godendo delle attenzioni della Repubblica di Venezia. Tutti - dal più grande al più piccolo - non mi risparmiavano la loro amicizia e benedicendomi gridavano “evviva!” (M.C. d'Arquien Sobieska a J.L. Sobieski, fuori Venezia verso Bologna, 6 II [1699], NGAB, f. 695, op. 1, n²58, k. 37v.). Questi momenti meravigliosi rimasero saldamente impressi nella memoria della sovrana a tal punto che durante il suo soggiorno a Roma, la regina considerò più volte l'idea di comprare un immobile a Venezia e di stabilirvi una sua residenza dove fermarsi durante il carnevale e poter incontrare i suoi figli (M.C. d'Arquien Sobieska a J.L. Sobieski, da Roma, 13 VIII [1701], NGAB, f. 695, op. 1, $\mathrm{n}^{\circ} 259$, k. 45v.-46r). Quest'idea nata nel 1701, fu concretizzata in un certo qual modo un anno dopo, quando la regina decise di incaricare Girolamo Alberti di fungere da intermediario per prendere in affitto un palazzo al Canal Grande o un casinò alla Riviera del Brenta (M.C. d'Arquien Sobieska a J.L. Sobieski, da Roma, 12 XI [1701], NGAB, f. 695, op. $1, \mathrm{n}^{\circ} 258, \mathrm{k} .97 \mathrm{v}$.) Il motivo di una tale decisione fu sempre il suo bisogno di vedere i figli: "È vero che penso che la situazione lì non sia molto sana ma sulla terra ferma ci sono delle case di villeggiatura per non stare sempre a Venezia" (Ella a esso, da Roma, 25 VI [1701], NGAB, f. 695 , op. $1, n^{\circ} 286$, k. 29r.). Si può presumere, anche se sono solo congetture non supportate dalle affermazioni della regina, che la sovrana volesse seguire da vicino il comportamento dell'imperatore Leopoldo I. Questi avrebbe dovuto assicurare al principe Jacopo un appannaggio lucrativo e prestigioso e di poter in ogni caso intervenire presso la corte di Vienna (M.C. d'Arquien Sobieska a J.L. Sobieski, Roma, 18 VI 1701, NGAB, f. 695, op. 1, n 272, k. 74v.; Ella a esso, Roma, 27 XI [1701], ibidem, k. 84r.; Ella a esso, Roma, 31 XII [1701], ibid., k. 89r.-89v.; Ella a esso, Roma, 25 II [1702], ibid., k. 94r.-97r.; Ella a esso, Roma, 
1 IV [1702], ibid., k. 110v.-112v; Skrzypietz, 2012, pp. 20-43). Maria Casimira non rinunciò mai al sogno di assicurare a uno dei suoi figli la corona del re di Polonia. Tuttavia, quest'idea non coincideva per niente con la realtà politica. La regina la menzionò in maniera dissimulata nelle sue lettere: "[...] Venezia la quale bisogna scegliere come sua sede, perché a Dio piacque governare in questo modo la sorte sfortunata della nostra famiglia" (M.C. d'Arquien Sobieska a J.L. Sobieski, Roma, 23 VII [1701], NGAB, f. 695, op. 1, n 286, k. 98r. cf. Ella a esso, Roma, 9 VII [1701], NGAB, f. 695, op. 1, n 277, k. 55v.).

I progetti della regina si realizzarono grazie a circostanze politiche mutevoli. Verso la fine del mese di febbraio del 1704, i principi Jacopo e Costantino furono rapiti sulla strada da Oława a Breslavia e in seguito furono imprigionati nella fortezza di Königstein per ordine di Augusto II. Lo stesso anno, Massimiliano II Emanuele perse il trono di Baviera, in seguito alla sconfitta di Höchstädt contro l'esercito imperiale. Dopo la sua partenza per Bruxelles e Versailles, sua Moglie Teresa Cunegonda Sobieska diventò reggente (Komaszyński, 1982a, pp. 84-100; Komaszyński, 1982b, pp. 555-573; Komaszyński, 1983, pp. 313-327). Tuttavia, il principe elettore progettava di far salire al potere sua suocera Maria Casimira d'Arquien Sobieska, con il passare del tempo. La stessa espresse il suo aggradimento per questi piani (Massimiliano II Emanuele di Baviera Wittelsbach a M.C. d'Arquien Sobieska, Bruxelles, $10 \mathrm{X}$ 1704, in: Archiv für österreichische geschichte, herausgegeben von der Österreichische Akademie der Wissenschaften, Wien 1848, pp. 384-386; esso a ella, Bruxelles, 14 XI 1704, in: ibid., pp. 388-389). Comunque, il viaggio intrapreso dalla sovrana verso la fine del 1704 attraverso Mantova, Loreto e Padova fu interrotto dalla cattiva notizia proveniente da Vienna: l'imperatore Leopoldo I non accordò il rilascio di un adeguato lasciapassare (Avvisi delle cose accadute in Roma negli anni 1703, 1704 e 1705, B.A.V., Cod. Ott. Lat. 2733, f. 22v.; M.C. d'Arquien Sobieska a Clemente XI, 13 I 1705, BL, Mss. Add. 8526, ff. 79-80; Ella a esso, Padova, 13 III 1705, ibid., ff. 81-84 e succ.; ella a Ferdinando Carlo Gonzaga, principe di Mantova, Roma, 21 I 1705, BOss, segn. 1468/II, p. 588; ella a esso, Padova, ibid., 24 V 1705, p. 590; Platania, 1989, pp. 165-168). Allora, Maria Casimira comprese di non potersi 
inoltrare ancor di più per insistere presso la famiglia Asburgo di intraprendere adeguate azioni contro l'elettore sassone né per supportare sua figlia. In questa situazione, senza l'esercito nel territorio bavarese e senza nessun consiglio del marito e dell'aristocrazia sulle questioni politiche, l'elettrice consorte decise di andare in Italia per incontrare la madre (Teresa Cunegonda Sobieska-Wittelsbach a M.C. d'Arquien Sobieska, [Monaco di Baviera], XII 1704, SM, GH KA, n 754, 1/9b). Nel febbraio dell'anno 1705, con la scusa di voler partecipare al carnevale, l'elettrice consorte si recò a Venezia passando per Graz e Salzsburg. L'11 marzo 1705, nella capitale della Serenissima Repubblica ebbe luogo l'incontro tra madre e figlia. Tuttavia, la gioia di questa riunione non durò a lungo. Teresa Cunegonda insistette per partire al più presto per la Baviera. Intanto sua madre sapeva che questa soluzione fu impossibile a causa dell'interdizione di attraversare le terre appartenenti agli Asburgo. Durante il soggiorno della madre e figlia a Venezia, ci furono tra loro diversi litigi riguardanti il cerimoniale. Maria Casimira fu indignata perché non immaginava che ci dovesse essere qualunque specie di protocollo tra due persone tanto vicine (M.C. d'Arquien a Teresa Cunegonda Sobieska-Wittelsbach, 30 III [1705], SM, GH KS, n 6644; ella a Ferdinando Carlo Gonzaga, principe di Mantova, Venezia, 2 IV1705, BOss, segn. 1468/II, p. 592). La regina Maria Casimira era indignata per il fatto che il diplomatico, il barone Scarlatti, avesse parlato a nome di sua figlia: "Non parlò d'altro che del torto che havevano havuto quelli che havevano qua rappresentanto, che ella n'havese usato verso la Sig. Elettrice con troppa gran superiorità offensiva del di lei carattere. Chiamo diversi de suoi per testimoni favorevoli delle sue assertive, tanto rispetto al Baldachino, allo Strato, al Casino, alla tavola, etc." (Giovanni Battista Scarlatii a Clemente XI, 26 V 1705, AAV, FAl., vol. 178, f. 70-71). Nel mese di maggio del 1705, la regina polacca lasciò Venezia (M.C. d'Arquien Sobieska a Massimiliano II Emanuele di Baviera, Roma, 2 i 9 VI 1705, SM, GH KA, n 754, 1/9b) e tornò a Roma. Intanto, sua figlia alla quale fu negato il passaggio per la Baviera, dovette aspettare, fedele come Penelope, suo marito il quale riprese il trono di Monaco di Baviera solo dieci anni dopo gli eventi di cui sopra (Komaszyński, 1982a, pp. 101-116; Schryver, 2003, pp. 165-180). 
I due soggiorni della regina Maria Casimira d'Arquien Sobieska a Venezia nel 1699 e nel 1705 furono nettamente diversi. Sicuramente fu il suo ruolo di regina a determinarlo. Infatti, durante la prima visita, Maria Casimira si presentò in veste ufficiale e venne accolta come testa coronata. La sua fama fu tanto grande quanto la gloria del suo defunto marito. Per questo motivo, tutti gli organi di vita pubblica, religiosa e culturale della Serenissima, incluse le città sotto il dominio della Repubblica, osservavano il cerimoniale per accoglierla nel modo adeguato.

Invece, il secondo soggiorno si svolse in un'atmosfera del tutto diversa: la regina fu trattata come un'ospite indesiderata. Tale situazione fu frutto delle circostanze influenzate dalla guerra di successione spagnola in corso e dal vantaggio dell'Impero che non voleva perdere la sua influenza in Baviera né interrompere le buone relazioni con l'elettore sassone. Per questo motivo, nel 1705, la vedova di Giovanni III non fu più accolta ufficialmente al Palazzo dei Dogi né furono dati grandi banchetti o feste in suo onore. Le autorità della Repubblica preferirono perfino non accogliere ufficialmente con tutti gli onori l'elettrice bavarese per non incappare nell'ira dell'imperatore Leopoldo I. Perciò, in quell'occasione anche Maria Casimira d'Arquien Sobieska venne trattata come un'ordinaria nobildonna polacca conformemente alla modalità incognito che essa stessa scelse di adottare durante il suo viaggio. Questa immagine abbastanza negativa della regina polacca derivava anche dai suoi malintesi con la figlia. Lo testimoniano anche ricchi archivi di documenti conservati riguardanti la visita della regina nel 1699 e scarsi ricordi relativi al viaggio di sei anni dopo, limitati praticamente solo alla corrispondenza privata.

Quello che indubbiamente affascinò la regina fu la vita musicale fiorente a Venezia. Grazie ad essa, la regina conobbe il meraviglioso e fiabesco mondo dell'opera di cui fu fervente patrona già durante il suo soggiorno nella Città Eterna. I drammi musicali diventarono opere di propaganda che cantavano la gloria della Casa Sobieski, le pretese dei figli della regina alla corona della Polonia e le aspirazioni della famiglia ad essere trattata come parte del sistema dinastico europeo (cf. Marku- 
szewska, 2012, passim; Markuszewska, 2013, pp. 187-191; Skrzypietz, 2010, pp. 189-209).

\section{BIBLIOGRAFIA}

\section{Manoscritti}

Archivio Apostolico Vaticano, Roma

- Fondo Avvisi: vol. 64

Archivio Storico Capitolino, Roma

- Archivio Capranica del Grillo, Fondo Scarlatti, n. 1187, 1190 (busta 4)

Archiwum Główne Akt Dawnych w Warszawie

- Archiwum Warszawskie Radziwiłłów, dz. III, sygn. 36

- Archiwum Publiczne Potockich, sygn. 51, 164

Biblioteca Apostolica Vaticana, Roma

- Fondo Albani: vol. 178

- Fondo Chigi: Cod. M.V.V.

- Fondo Ottoboni: Cod. Ott. Lat. 2733

British Library London, Mss. Add. 8526

Nacyanalny Gistaryčny Archiŭ Belarusi w Mińsku, f. 695, op. 1, n² 241, 258, 259, 260, 272, 277, 286

Staatsarchiv München, Geheimes Hausarchiv

- Kasten Schwrzt: n 6644

- Korrespondenz Akt: $\mathrm{n}^{\circ} 754,1 / 9 \mathrm{~b}$

Zakład Narodowy im. Ossolińskich we Wrocławiu: 1468/II

\section{Stampe vecchie}

Bassani, A. (1700). Viaggio a Roma della Sua Reale M[aest]à di Maria Casimira, Regina di Polonia, vedova dell'Invittissimo Giovanni III. Roma: Barberini.

Bernardi, F. (1699). Viaggio a Roma della Sacra Reale Maestà di Maria Casimira di Polonia. Firenze: Biblioteca provinciale dei Cappuccini.

Biancolini, G. et al. (1750). Notizie storiche delle chiese di Verona raccolte da [...], vol. 1. Verona: Agostino Carattoni Stampator Vescovile.

Rovere, S. (1704). Vita del serenissimo prencipe Silvestro Valiero doge di Venetia. Scritta dal padre d. Silvestro Rovere. Venezia: Stamparia Antonio Bortoli. 


\section{Edizioni originali}

Archiv für österreichische Geschichte (1848). Wien: Österreichische Akademie der Wissenschaften.

Ferrari, G. (1868). Relazione storica del passaggio, e della breve dimora nella città di Padova della Principessa Reale di Polonia e Regina Maria Amalia moglie di Carlo Re delle Due Sicilie di Girolamo Ferrari Dottor. 1738. In S. Krzyżanowski (Ed.), Wspomnienia z Padwy. Notatki z podróży (pp. 31-60). Kraków: Drukarnia "Czasu” W. Kirchmayera.

Sobieski, J. (1860). Listy Jana Sobieskiego do żony Marii Kazimiery wraz $z$ listami innych znakomitych osób przez Jerzego Samuela Bandtkiego z oryginatu niegdyś archiwum Sobieskich przepisane (edited by A.Z. Helcel). Kraków: Drukarnia C.K. Uniwersytetu Jagiellońskiego.

\section{Letteratura}

Bély, L. (1999). La Société des princes: XVI $-X V I I I^{e}$ siècle. Paris: Fayard. Cavazza, S. (2007). Marco d'Aviano. In Dizionario Biografico degli Italiani, vol. 69. Retrieved from https://www.treccani.it/enciclopedia/marco-daviano_(Dizionario-Biografico)/

Conforti, G. (1988-1989/1989-1990). Villa Nichesola Mocenigo a Ponton di Sant'Ambrogio. Annuario Storico della Valpolicella, 7, 65-124.

Duron, J. (Ed.). (2010). Le carnaval de Venise, 1699 : d'André Campra et Jean-François Regnard. Livret, études et commentaires. Wavre: Mardaga.

Jačov, M. (2003). Europa i Osmanie w okresie Lig Świętych. Polska między Wschodem a Zachodem. Kraków: Polska Akademia Umiejętności.

Komaszyński, M. (1982a). Teresa Kunegunda Sobieska. Warszawa: Państwowy Instytut Wydawniczy.

Komaszyński, M. (1982b). Die politische Rolle der bayerischen Kurfürstin Theresia Kunigunde. Zeitschrift für bayerische Landesgeschichte, 45, 3, 555-573.

Komaszyński, M. (1983). Die Beziehungen zwischen den Höfen der Wittelsbacher und dem von Sobieski in der zweiten Hälfte des XVII. Jahrhunderts. Zeitschrift für bayerische Landesgeschichte, 46, 2, 313-327.

Komaszyński, M. (1984). Maria Kazimiera d'Arquien Sobieska królowa Polski (1641-1716). Kraków: Wydawnictwo Literackie. 
Komaszyński, M. (1988). Il viaggio trionfale di una regina di Polonia in Italia. In E. Kanceff, \& R. Lewanski (Eds.), Viaggiatori polacchi in Italia (pp. 153-163). Genève: Slatkine.

Komaszyński, M. (1990). Ostatnia kampania Jana Sobieskiego w relacjach posła weneckiego Girolamo Albertiego. In R.C. Levanski, \& S. de Fanti (Eds.), Munera Polonica et Slavica (pp. 109-115). Udine: Istituto di Lingue e Letterature dell'Europa Orientale, Università degli Studi di Udine.

Langen, U. (2002). The Meaning of Incognito. The Court Historian, 2, $145-155$.

Lewański, R.K. (1984). La reine Marie Casimire d'Arquien Sobieska de passage à Verone. In E. Kanceff (Ed.), Voyageurs français à Verona (pp. 27-32). Genève: Slatkine.

Markuszewska, A. (2010). Festa i muzyka na dworze Marii Kazimiery Sobieskiej w Rzymie (1699-1714). Warszawa: Muzeum Pałacu Króla Jana III w Wilanowie.

Markuszewska, A. (2013). Muzyka ku chwale Jana III na rzymskim dworze Marii Kazimiery Sobieskiej. In D. Walawender-Musz (Ed.), Primus inter pares. Pierwszy pośród równych czyli opowieść o królu Janie III Sobieskim (pp. 187-191). Warszawa: Muzeum Pałacu Króla Jana III w Wilanowie.

Pietrzak, J. (2018). L'ingresso di Maria Casimira d'Arquien Sobieska a Roma nel 1699 e la sua cornice cerimoniale. In J.A. Chrościcki, Z. Flisowska, \& P. Migasiewicz (Eds.), I Sobieski a Roma. La famiglia reale polacca nella Città Eterna (pp. 70-88). Warszawa: Muzeum Pałacu Króla Jana III w Wilanowie.

Platania, G. (1986). Arrivo a Venezia e visita a Marco d'Aviano di Maria de la Grange d'Arquien regina di Polonia. Quaderni Utinensi, IV, 7-8, 11-23.

Platania, G. (1988). Viaggio in Italia di una dama polacca: Maria Casimira Sobieska. In E. Kanceff, \& R. Lewanski (Eds.), Viaggiatori polacchi in Italia (pp.165-181). Genève: Slatkine.

Platania, G. (1989). Gli ultimi Sobieski e Roma. Fasti e miserie di una famiglia reale polacca tra Sei e Settecento (1699-1715). Roma: Vecchiarelli.

Platania, G. (1998). Diplomazia e guerra turca nel XVII secolo. La politica diplomatica polacca e la "lunga guerra turca" (1673-1683). In G. Motta (Ed.), I Turchi, il Mediterraneo e l'Europa (pp. 242-268). Milano: FrancoAngeli. 
Platania, G. (2010). Mamma li turchi! L'idea di crociata nell'età moderna. Viterbo: Sette Città.

Schryver, R. de (2003). Princess Teresa Kunegunda Sobieska 1676-1730. Thirty-five years of solitude. In T. Soldatjenkova, \& E. Waegemans (Eds.), For East is East: Liber Amicorum Wojciech Skalmowski (pp. 165-180). Leuven-Paris-Dudley: Peeters and Departement Oosterse Studies.

Selfridge-Field, E. (2007). The Calendar of Venetian Opera. A New Chronology of Venetian Operas and Related Genres, 1660-1760. Stanford: Stanford University Press.

Setton, Kenneth M. (1991). Venice, Austria and the Turks in the Seventeenth Century. Philadelphia: American Philosophical Society.

Simonato, R. (Ed.). (1993). Marco d'Aviano e il suo tempo: un cappuccino del Seicento, gli Ottomani e l'Impero. Atti del convegno storico internazionale Pordenone 12-13 novembre 1993. Pordenone: Concordia sette.

Skrzypietz, A. (2010). Propagandowe wykorzystanie wiktorii wiedeńskiej w polityce dynastycznej Sobieskich. In S. Achremczyk (Ed.), Między Barokiem a Oświeceniem: wielkie bitwy (pp. 189-209). Olsztyn: Ośrodek Badań Naukowych im. W. Kętrzyńskiego w Olsztynie.

Skrzypietz, A. (2011). Królewscy synowie - Jakub, Aleksander i Konstanty Sobiescy. Katowice: Wydawnictwo Uniwersytetu Śląskiego.

Skrzypietz, A. (2012). Królewicz Jakub Sobieski i jego kontakty z dworami europejskimi do wybuchu wielkiej wojny północnej. Wieki Stare Nowe, 4, 20-43.

Tassini, G. (1872). Curiosità veneziane ovvero origini delle denominazioni stradali di Venezia. Venezia: Grimaldo.

Urban, L. (2007). Banchetti veneziani dal Rinascimento al 1797. Venezia: Fondazione Cini.

Wójcik, Z. (1976). Rzeczpospolita wobec Turcji i Rosji 1674-1679. Studium $z$ dziejów polskiej polityki zagranicznej. Wrocław: Ossolineum.

Wrana, M. (2019). Wizyty królewskie u grobu św. Antoniego (XVI-XVIII w.). In M. Lenart, M. Wrana, \& M. Wojtkowska-Maksymik (Eds.), Polacy przy grobie św. Antoniego w Padwie. Pamiątki, donacje, świadectwa kultu (wieki XVI-XIX), cz. I (pp. 337-342). Opole: Wydawnictwo Uniwersytetu Opolskiego.

\section{Abbreviazioni}

AAV - Archivio Apostolico Vaticano, Roma 


$\begin{array}{ll}\text { AGAD } & \text { - Archiwum Główne Akt Dawnych w Warszawie } \\ \text { APP } & \text { - Archiwum Publiczne Potockich (in: Archiwum Główne Akt } \\ & \text { Dawnych w Warszawie). } \\ \text { AR } & \begin{array}{l}\text { - Archiwum Warszawskie Radziwiłłów (in: Archiwum Głów- } \\ \text { ne Akt Dawnych w Warszawie). }\end{array} \\ \text { ARC } & \text { - Archivio Storico Capitolino, Roma } \\ \text { ACS } & \text { - Archivio Capranica del Grillo, Fondo Scarlatti (in: Archivio } \\ & \text { Storico Capitolino, Roma) } \\ \text { B.A.V } & \text { - Biblioteca Apostolica Vaticana, Roma } \\ \text { BL } & \text { - British Library w Londynie } \\ \text { BOss } & \text { - Zakład Narodowy im. Ossolińskich we Wrocławiu } \\ \text { BUW } & \text { - Biblioteka Uniwersytetu Warszawskiego w Warszawie } \\ \text { FA } & \text { - Fondo Avvisi (in: Archivio Apostolico Vaticano, Roma) } \\ \text { FAl. } & \text { - Fondo Albani (in: Archivio Apostolico Vaticano, Roma) } \\ \text { FCh } & \text { - Fondo Chigi (in: Biblioteca Apostolica Vaticana) } \\ \text { GH KA } & \text { - Geheimes Hausarchiv Korrespondenz Akt (in: Staatsarchiv } \\ & \text { München) } \\ \text { KS } & \text { - Kasten Schwarz } \\ \text { NGAB } & \text { - Nacyanalny Gistaryčny Archiŭ Belarusi w Mińsku } \\ \text { SM } & - \text { Staatsarchiv München }\end{array}$

Riassunto: La regina Maria Casimira d'Arquien Sobieska ebbe molte relazioni con Venezia. Durante la vita di Giovanni III Sobieski mantenne contatti con i diplomatici Angelo Morosini e Girolamo Alberti. Partita dalla campagna nell'ottobre 1698 per la Città Eterna, dopo aver varcato il confine dell'Impero con l'Italia, la regina incontrò le principali città del Veneto, successivamente Verona, Padova e Venezia. Il suo secondo soggiorno nella Repubblica di Venezia ebbe luogo nel 1705, quando l'ex-sovrana, con il permesso papale, andò a incontrare la figlia Teresa Kunegunda, elettrice di Baviera. Nell'articolo si trattano diversi aspetti dei viaggi della regina, oltre a questioni politiche. In primo luogo, si presta attenzione alla cerimonia di ricevimento della regina-vedova da parte delle autorità della Serenissima, rappresentanti dell'élite veneziana e inviati di paesi stranieri residenti a Venezia. Successivamente, si richiama l'attenzione sui luoghi visitati dalla regina, sulla pietà da lei manifestata, e si presentano le osservazioni relative alla vita cittadina formulate direttamente da Maria Casimira e dai suoi cortigiani, anche in relazione a Verona e Padova. Alla fine si sottolinea l'interesse della regina per il repertorio drammatico e musicale presentato sui palcoscenici veneziani e per tutta la vita culturale, compreso il carnevale.

Parole chiave: Veneto, Regina Maria Casimira Sobieska, viaggio, carnevale, regole cerimoniali 